\title{
Substrate-induced bulk alignment of liquid crystals
}

\section{Zhang, Zhengping; Chakrabarti, A.; Mouritsen, Ole G.; Zuckermann, Martin}

Published in:

Physical Review E. Statistical, Nonlinear, and Soft Matter Physics

Link to article, DOI:

10.1103/PhysRevE.53.2461

Publication date:

1996

Document Version

Publisher's PDF, also known as Version of record

Link back to DTU Orbit

Citation (APA):

Zhang, Z., Chakrabarti, A., Mouritsen, O. G., \& Zuckermann, M. (1996). Substrate-induced bulk alignment of liquid crystals. Physical Review E. Statistical, Nonlinear, and Soft Matter Physics, 53(3), 2461-2465.

https://doi.org/10.1103/PhysRevE.53.2461

\section{General rights}

Copyright and moral rights for the publications made accessible in the public portal are retained by the authors and/or other copyright owners and it is a condition of accessing publications that users recognise and abide by the legal requirements associated with these rights.

- Users may download and print one copy of any publication from the public portal for the purpose of private study or research.

- You may not further distribute the material or use it for any profit-making activity or commercial gain

- You may freely distribute the URL identifying the publication in the public portal 


\title{
Substrate-induced bulk alignment of liquid crystals
}

\author{
Zhengping Zhang, ${ }^{1, *}$ Amitabha Chakrabarti, ${ }^{1}$ Ole G. Mouritsen, ${ }^{2}$ and Martin J. Zuckermann ${ }^{3}$ \\ ${ }^{1}$ Department of Physics, Kansas State University, Manhattan, Kansas 66506 \\ ${ }^{2}$ Department of Physical Chemistry, The Technical University of Denmark, DK-2800 Lyngby, Denmark \\ ${ }^{3}$ Department of Physics, McGill University, Montréal, Québec, Canada H3A 2T8
}

(Received 17 July 1995)

\begin{abstract}
The Gay-Berne model for liquid crystals in the presence of a substrate surface is studied using the hybrid Monte Carlo method. A simple non-mean-field substrate-molecule potential is proposed to describe the effects of rubbed polymer-coated substrates on the liquid crystals. Effects of the substrate surface on the bulk alignment of the liquid crystals are studied. It is found that the bulk pretilt angle is controlled by the surface through the orientation of the adsorbed liquid crystal monolayer. This is consistent with the results of recent experimental studies.
\end{abstract}

PACS number(s): $61.30 . \mathrm{Gd}, 61.30 . \mathrm{Cz}, 68.45 . \mathrm{Da}$

Surface-induced bulk alignment of nematic liquid crystals is of great importance to liquid crystal display technology and has therefore attracted considerable interest from experimentalists in the past few years [1]. Recent experiments show that the orientational distribution of the liquid crystal monolayer close to the substrate surface completely determines the nematic bulk alignment $[2,3]$. However, the manner in which the structure of the substrate surface and its microscopic interactions with liquid crystal molecules are responsible for the phenomenon is as yet unknown. The Landau-de Gennes formalism has been used for the analysis of the relevant experiment data, but this is a phenomenological approach and involves many macroscopic material parameters. Furthermore, in this approach, a priori assumptions concerning the orientational distribution function of liquid crystal molecules in the monolayer close to the substrate surface are usually required [3]. Several mean-field theories based on molecular models have also been proposed to explain the surface-related phenomena of the liquid crystals [1], but none of these is closely related to the relevant experiments. The main problem lies in checking the validity of the assumed coupling between the rotational and translational degrees of freedom of the molecules in the vicinity of the surface, as this is an essential ingredient of the mean-field theories [4]. An understanding of the nature of the microscopic interactions responsible for the nematic bulk alignment therefore requires the development of a microscopic model, which is reasonably realistic and yet sufficiently tractable for use in numerical simulations.

In this paper we determine the dependence of the bulk orientation of liquid crystals on substrate-molecule interactions based on a hybrid Monte Carlo simulation

\footnotetext{
${ }^{*}$ Present address: Department of Polymer Engineering, College of Polymer Science and Polymer Engineering, The University of Akron, Akron, $\mathrm{OH} 44325-0301$.
}

study of an extended Gay-Berne model in the presence of substrate surfaces. The conventional Gay-Berne (GB) model [5] is a reasonably realistic model for the behavior of liquid crystals in the bulk and it provides a description of the various bulk phases, i.e., vapor, isotropic liquid, nematic, and smectic mesophases [6]. It was, however, difficult to make an accurate study of the equilibrium behavior of the high-density mesophases using this model [6] before Zhang and Chakrabarti applied the hybrid Monte Carlo method [7] to the model [8]. The GB model had already been used before in the work of Chalam et al. [6] to study the liquid crystals confined in a pore with parallel, homeotropic surfaces. These studies used a mean-field approximation for the interaction between the substrate surface and the liquid crystal molecules (substrate-molecule potential) in which the orientational and translational degrees of freedom of the molecules were separated artificially, since the geometric shape of the molecules was not properly taken into account. In this paper we propose a form for the substratemolecule potential in the spirit of the GB model. The purpose of the present work is to show how this affects the phase of the bulk liquid crystalline molecules.

The GB potential was originally proposed as a numerical fit to a four-site Lennard-Jones model [5] and it was shown to give a reasonable account of both the anisotropic repulsive and attractive forces. The GB model therefore plays a similar important role in the studies of the liquid crystals to that the Lennard-Jones potential in simple fluids. Furthermore, the GB potential reduces to the Lennard-Jones potential in the isotropic limit. The GB potential is given by [5]

$$
U_{i j}=4 \epsilon_{i j}\left\{\left[\frac{\sigma_{0}}{r_{i j}-\sigma_{i j}+\sigma_{0}}\right]^{12}-\left[\frac{\sigma_{0}}{r_{i j}-\sigma_{i j}+\sigma_{0}}\right]^{6}\right\}
$$

where $\epsilon_{i j}$ and $\sigma_{i j}$ are the coupling constant and interaction range, respectively. Both parameters are depen- 
dent on the orientations of the $i$ th and $j$ th molecules and $r_{i j}=\left|\mathbf{r}_{i}-\mathbf{r}_{j}\right|$ is the distance between their centers of mass given by $\mathbf{r}_{i}$ and $\mathbf{r}_{j} . \epsilon_{i j}$ and $\sigma_{i j}$ are defined as $\epsilon_{i j}=\epsilon_{0}\left[1-\psi^{2}\left(\hat{\mathbf{e}}_{i} \cdot \hat{\mathbf{e}}_{j}\right)^{2}\right]^{-1 / 2} \Omega_{i j}^{2}\left(\psi^{\prime}\right)$ and $\sigma_{i j}=\epsilon_{0} \Omega_{i j}^{-1 / 2}(\psi)$, respectively, with $\Omega_{i j}(x)=1-\frac{x}{2}\left[\left(\hat{\mathbf{r}}_{i j} \cdot \hat{\mathbf{e}}_{i}+\hat{\mathbf{r}}_{i j} \cdot \hat{\mathbf{e}}_{j}\right)^{2} /(1+\right.$ $\left.\left.x \hat{\mathbf{e}}_{i} \cdot \hat{\mathbf{e}}_{j}\right)+\left(\hat{\mathbf{r}}_{i j} \cdot \hat{\mathbf{e}}_{i}-\hat{\mathbf{r}}_{i j} \cdot \hat{\mathbf{e}}_{j}\right)^{2} /\left(1-x \hat{\mathbf{e}}_{i} \cdot \hat{\mathbf{e}}_{j}\right)\right] . \quad \hat{\mathbf{e}}_{i}$ is the axial vector of the $i$ th molecule and $\hat{\mathbf{r}}_{i j}$ is the unit vector along $\mathbf{r}_{i}-\mathbf{r}_{j}$. The parameter $\psi$ is related to the anisotropy in the molecular shape and is given by $\psi=\left[\left(\sigma_{e e} / \sigma_{s s}\right)^{2}-1\right] /\left[\left(\sigma_{e e} / \sigma_{s s}\right)^{2}+1\right]$, where $\sigma_{e e} / \sigma_{s s}$ is the ratio of the range parameters for the end-to-end and sideby-side configurations, i.e., the ratio of molecular length to breadth. Similarly, the parameter $\psi^{\prime}$ is the ratio of the well depths of the GB potential for the configurations and is given by $\psi^{\prime}=\left[1-\left(\epsilon_{e e} / \epsilon_{s s}\right)^{1 / 2}\right] /\left[1+\left(\epsilon_{e e} / \epsilon_{s s}\right)^{1 / 2}\right]$.

We propose the following form for the substratemolecule (SM) potential, which takes into account the shape of the GB molecules

$$
U_{w i}=\epsilon_{w i}\left\{\left[\frac{\sigma_{w i}}{z_{i}}\right]^{9}-\left[\frac{\sigma_{w i}}{z_{i}}\right]^{3}\right\}-\operatorname{sgn}\left(\hat{\mathbf{e}}_{i} \cdot \hat{\mathbf{z}}\right) \mathbf{d}_{1} \cdot \hat{\mathbf{e}}_{i},
$$

where $z_{i}$ is the distance of the $i$ th molecule from the substrate surface, $\operatorname{sgn}(x)$ is the sign function, and $\mathbf{d}_{1}$ is a vector lying in the surface. The first term is a modification of the Steele-Hasley surface-gas potential $[9,6]$ with the coupling constant $\epsilon_{w i}$ depending on the orientation of the $i$ th molecule: $\epsilon_{w i}=\epsilon_{w}\left[1-\psi_{w}^{\prime}\left(\hat{\mathbf{e}}_{i} \cdot \hat{\mathbf{z}}\right)^{2}\right]^{2}$. Here $\epsilon_{w}$ is a constant, $\hat{\mathbf{z}}$ is the unit vector normal to the surface, and $\psi_{w}^{\prime}=1-\left(\epsilon_{w \perp} / \epsilon_{w \|}\right)^{1 / 2}$. The range parameter $\sigma_{w i}=\sigma_{w}\left[1-\psi_{w}\left(\hat{\mathbf{e}}_{i} \cdot \hat{\mathbf{z}}\right)^{2}\right]^{-1 / 2}$, where $\sigma_{w}$ is a constant and $\psi_{w}=1-\left(\sigma_{w \|} / \sigma_{w \perp}\right)^{2}$. The subscripts $\|$ and $\perp$ denote the corresponding values when the molecules are either parallel or perpendicular to the surface. The second term describes a "rubbed" surface, i.e., a surface that has been treated in such a way as to orient molecules close to the surface in a specific direction $\mathbf{d}_{1}$. Such molecules are defined in our model as molecules for which $z_{i}<R_{a d}$. Here $R_{a d}$ is a short-range parameter such that $R_{a d}<1.5 \sigma_{0}$. This gives a tractable phenomenological description of rubbing effects rather than full molecular modeling because of the complexity of the microscopic interactions involved. The rubbing term, however, has the correct symmetries of oppositely rubbed parallel surfaces as in the experimental situation [3].

The Hamiltonian describing our model system is then

$$
\mathcal{H}=\frac{1}{2 m} \sum_{i} \mathbf{p}_{i}{ }^{2}+\frac{1}{2 I} \sum_{i}{\mathbf{l}_{i}}^{2}+\sum_{i<j} U_{i j}+\sum_{i} U_{w i},
$$

where $\mathbf{p}_{i}$ and $\mathbf{l}_{i}$ are the momentum and the angular momentum of the $i$ th molecule, respectively. The mass $m$ of the GB molecules is used as unity in our simulations. We further assume, for simplicity, that the moment of inertia $I$ of each molecule is also unity since the density distribution of GB molecules is unspecified. In this work, we have used the hybrid Monte Carlo (HMC) simulation method [7]. This method was recently applied to bulk liquid crystals described by the GB model [8]. The HMC scheme is a combination of a molecular dynamics (MD) simulation and a Monte Carlo (MC) algorithm and it retains the advantages of both techniques. The simulations proceed as follows. Starting from the current configuration $\{\mathbf{r}, \hat{\mathbf{e}}\}$, we first generate randomly new momenta and angular momenta $\{\mathbf{p}, \mathbf{l}\}$ according to a MaxwellBoltzmann distribution at a finite temperature $T$. We then integrate the equations of motion associated with the Hamiltonian of Eq. (3) for $N_{\mathrm{MD}}$ time steps of length $\delta t_{\mathrm{MD}}$ using the leapfrog time integration algorithms for both translational and orientational degrees of freedom [10]. In this way a possible new configuration $\left\{\mathbf{r}^{\prime}, \hat{\mathbf{e}}^{\prime}\right\}$ and new translational and angular momenta $\left\{\mathbf{p}^{\prime}, \mathbf{l}^{\prime}\right\}$ are obtained. The new configuration is accepted with probability $P_{A}=\min \{1, \exp (-\beta \Delta \mathcal{H})\}$, where $\beta=1 / k_{B} T$ and $\Delta \mathcal{H}=\mathcal{H}\left(\left\{\mathbf{p}^{\prime}, \mathbf{l}^{\prime}, \mathbf{r}^{\prime}, \hat{\mathbf{e}}^{\prime}\right\}\right)-\mathcal{H}(\{\mathbf{p}, \mathbf{l}, \mathbf{r}, \hat{\mathbf{e}}\})$. Regardless of whether the new configuration has been accepted or not, the momenta and the angular momenta are refreshed after every MC acceptance decision in order to satisfy the detailed balance condition [7], thereby generating the equilibrium distribution $P_{\text {eq }} \sim \exp \left\{-\beta\left(\sum_{i<j} U_{i j}+\right.\right.$ $\left.\left.\sum_{i} U_{w i}\right)\right\}$ in $\{\mathbf{r}, \hat{\mathbf{e}}\}$ space.

The molecular anisotropy and the well depth ratio parameters in the GB potential are chosen to be $\sigma_{e e} / \sigma_{s s}=$ 3 and $\epsilon_{e e} / \epsilon_{s s}=0.2$, respectively, as in previous studies $[5,6,8]$. The simulations are carried out at constant volume with a reduced number density $\rho^{*}=N \sigma_{0}{ }^{3} / V=$ 0.3 at a reduced temperature $T^{*}=1.0$, in units of $\epsilon_{0}$, where $N$ is the total number of molecules in the system of volume $V$. The bulk system is in the nematic phase for these parameters [8]. The molecular anisotropy parameters of the SM potential are chosen to be similar to those of the GB potential for most cases, i.e., $\sigma_{w \|} / \sigma_{w \perp}=1 / 3$ and $\epsilon_{w \perp} / \epsilon_{w \|}=1 / 5$, although parameters for stronger anisotropy are also used. The basic simulation box is rectangular with the three sides of length $L_{x}, L_{y}$, and $L_{z}$, which forms a convenient coordinate system for the molecules. The values $L_{x}=L_{y}=13.5 \sigma_{0}$ and $L_{z}=13.5 \sim 22.5 \sigma_{0}$ were used in our simulations. In the $z$ direction, the two surfaces at $z=-L_{z} / 2$ and $+L_{z} / 2$ are defined to be the planes where $U_{w i}$ becomes infinite. Periodic boundary conditions are only applied in the $x$ and $y$ directions. In our simulations, all the potentials are truncated at $r_{c} \approx 4.0 \sigma_{0}$. All other details of the simulations are the same as those reported in Ref. [8].

In Fig. 1 we present the number density profiles in the $z$ direction obtained for different values of the parameters $\epsilon_{w}, \sigma_{w}, \sigma_{w \|} / \sigma_{w \perp}$, and $\epsilon_{w \perp} / \epsilon_{w \|}$ in the absence of rubbing, i.e., $d_{1}=0$. It is a common feature of such equilibrium profiles to exhibit structure in the form of high-density peaks close to the surfaces [11] with a flat density profile in the bulk with the density given by its unperturbed average value $\rho_{\text {av }} \approx \rho^{*}=0.3$. The peaks represent molecular monolayers and the structureless density profile observed in the bulk indicates that a smectic phase [6] has not been induced by the presence of substrate surfaces at $T^{*}=1.0$ and $\rho^{*}=0.3$. The values of the parameters in Fig. 1 are $\sigma_{w}=0.5 \sigma_{0}, \sigma_{w \|} / \sigma_{w \perp}=1 / 3$, and $\epsilon_{w \perp} / \epsilon_{w \|}=1 / 5$ and the symbols circle, square, and diamond correspond to $\epsilon_{w}=0.5 \epsilon_{w}^{0}, \epsilon_{w}^{0}$, and $2 \epsilon_{w}^{0}$, respec- 


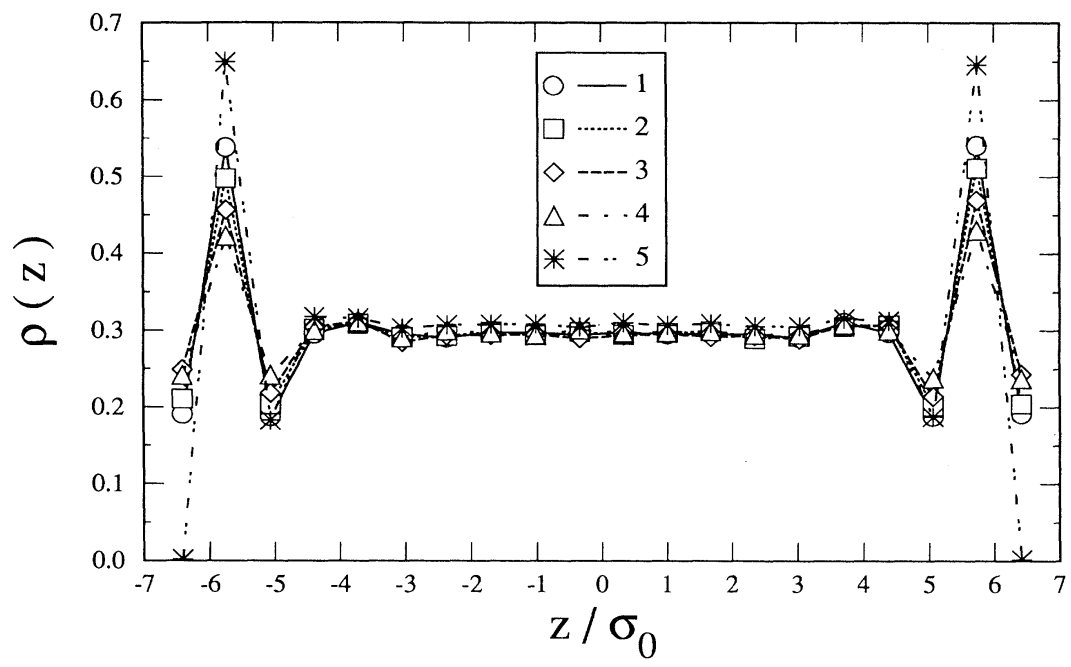

FIG. 1. Number density profiles $\rho(z)$ in the $z$ direction at $T^{*}=1.0$ and $\rho^{*}=0.3$ for the different cases with the parameters of groups 1-5. $\quad \mathbf{d}_{1}=0$. $L_{x}=L_{y}=L_{z}=13.5 \sigma_{0}$.

tively. Here $\epsilon_{w}^{0}=5 \sqrt{3} \epsilon_{0}$ is chosen so that the well depth of the SM potential for molecules oriented parallel to the surface is twice as large as that of the GB potential. Increasing the value of $\epsilon_{w}$ results in an increase in the local density close to the surfaces and a decrease in the highdensity peaks (i.e., monolayers), as the stronger SM interactions favor a movement of the molecules towards the surfaces. As a consequence of the decrease in the density of the monolayers, the density of the "wells" increases due to the weaker repulsive interactions of the molecules in the monolayers. Similar changes are observed in the case with $\sigma_{w \|} / \sigma_{w \perp}=1 / 5, \epsilon_{w \perp} / \epsilon_{w \|}=1 / 9$, and $\epsilon_{w}=\epsilon_{w}^{0}$, as shown by the data represented by $\triangle$ in Fig. 1 . The molecules parallel to the surface are energetically more favored due to the enhanced anisotropy in the SM potential. The value of the parameter $\sigma_{w}$ was found to affect the density close to the surfaces strongly but in a way opposite that observed in the above cases. The choice of $\sigma_{w}=0.7 \sigma_{0}$, while keeping the other parameters the same as those in the case given by circles in Fig. 1, results in an increase in the density of the monolayers and a decrease at their "shoulders," as shown by the data represented by the symbol asterisks in Fig. 1. This can be understood as follows. The repulsive interactions of the substrate on the molecules close to the surface become stronger due to the increase in $\sigma_{w}$ and the attractive interactions are correspondingly weakened in this range. The molecular density adjacent to the surface therefore decreases considerably, resulting in a density increase in the monolayers and slightly in the bulk. Furthermore, the density of the "wells" decreases due to the stronger repulsive interactions due to the molecules in the monolayers.

Figure 2 shows the nematic order parameter profiles $Q(z)$ corresponding to all the cases in Fig. 1. $Q(z)$ is evaluated as the largest eigenvalue of a second-rank tensor $Q_{\alpha \beta}(z)=\sum_{\left|z_{i}-z\right|<\delta} \frac{1}{2}\left(3 e_{i \alpha} e_{i \beta}-\delta_{\alpha \beta}\right)$, where $\alpha \beta=x, y, z$ and $e_{i \alpha}$ is the $\alpha$ th component of $\hat{\mathbf{e}}_{i}$ for the $i$ th molecule. The order parameter profiles $Q(z)$ are consistent with the density profiles $\rho(z)$ (see Fig. 1), although the influence of fluctuations due to the finite size of the system under study [12] can directly be seen in the order parameter

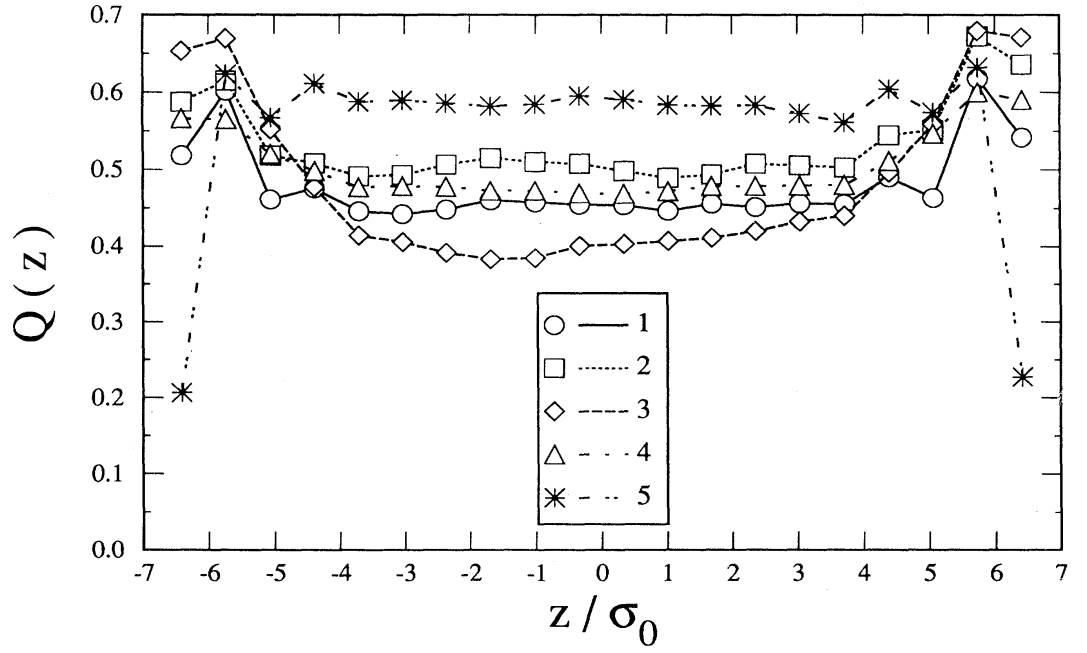

FIG. 2. Nematic order parameter profiles $Q(z)$ in the $z$ direction corresponding to all the cases in Fig. $1 . \mathbf{d}_{1}=\mathbf{0}$. $L_{x}=L_{y}=L_{z}=13.5 \sigma_{0}$. 
measurements. The nematic order of the molecules close to the surfaces is higher than that in the bulk for most cases because of the SM potential and the higher densities close to the surface. For all the cases, the nematic order parameter in the bulk is found to be in the range of 0.4-0.6, consistent with the values of the order parameter observed experimentally in nematic phases [13].

Figure 3 gives profiles for the average tilt angle $\alpha(z)$ in the $z$ direction for all the cases studied in Fig. 1. $\alpha(z)$ is related to the average orientation of the molecular alignment at the position $z$ described by the unit eigenvector $\hat{\mathbf{n}}(z)$ of $Q_{\alpha \beta}(z)$ corresponding to $Q(z)$ by $\alpha(z)=90^{\circ}-\arccos [|\hat{\mathbf{n}}(z) \cdot \hat{\mathbf{z}}|]$. Figure 3 shows that the bulk molecules are all tilted at an angle, known as the pretilt angle, with respect to the surfaces. In our model system the pretilt angle is found to vary over a range of about $15^{\circ}-25^{\circ}$, corresponding to the changes in the SM potential. The data shown in Fig. 3 suggest the following mechanism for the existence of a pretilt angle. The SM potential directly affects the orientations of the molecules in the monolayers, which then readjust the bulk pretilt angle. The larger the tilt angle of the monolayers, the larger the bulk pretilt angle. The obvious difference between the two angles is probably due to the presence of the low-density "wells." This weakens the correlations between the two angles. The molecular orientation of the monolayers results from a competition between the SM potential and the intermolecular interactions. To be better accommodated, the molecules in the monolayers tend to lie parallel to each other due to the intermolecular interactions and perpendicular to the surface while the SM potential favors the molecules in the monolayers to be parallel to the surfaces. This is consistent with experimental observations for the tilt of the anchoring directions of the 4 - $n$-octyl- $4^{\prime}$-cyanobiphenyl (8CB) molecules on most polyimide films. In fact, the molecules in the first monolayer make an angle of approximately $70^{\circ}$ with the surface normal (i.e., $\alpha \approx 20^{\circ}$ ), while the anchoring direction is planar $\left(\alpha \approx 0^{\circ}\right)$ on the polyimide films $[14,1]$.

Based on the above proposal for the mechanism by which the pretilt angle can be determined, one may ex- pect that the pretilt angle can be reduced by weakening the intermolecular attractive interactions in the monolayers. We find that this is indeed the case. The data shown by the pluses in Fig. 3 corresponds to the case denoted by the asterisks, except for a lower value of intermolecular coupling constant $\sqrt{\varepsilon_{i} \varepsilon_{j}}$ between the $i$ th and $j$ th molecules in the vicinity of either surface. Here $\varepsilon_{i}=0.1$ if the distance of the $i$ th molecule to the surface is less than $R_{a d}$; otherwise $\varepsilon_{i}=1$. The pretilt angle is evidently reduced in this case. This could represent a realistic situation since the intermolecular interactions in the monolayers might be weakened due to redistribution of the molecular charge close to the surfaces [15].

Figure 4 shows the deviations of the tilt angles from their previous values under the influence of oppositely rubbed surfaces. These were obtained for the cases denoted by the asterisks and pluses, respectively, in Fig. 3. The deviation is defined as the difference in the value of $\alpha(z)$ between the presence and the absence of rubbing, namely, $\delta \alpha(z)=\alpha_{\mathbf{d}_{1}}(z)-\alpha_{\mathbf{0}}(z)$. Without loss of generality, we chose the $x$ direction to be the direction of rubbing so that $\mathbf{d}_{1}=d_{1} \hat{\mathbf{x}}$, where $d_{1}$ is the rubbing strength. The data presented in Fig. 4 indicate that the effect of the rubbing results in a reduction of the pretilt angle. The effect is more significant at lower temperatures for the same rubbing strength as the thermal fluctuations are suppressed at these temperatures [16].

We have shown, using a basic model for the SM potential, that substrate rubbing has a significant effect on the pretilt angle. Let us now compare our findings with experimental results. It is found in recent experiments [3] that the pretilt angle increases with increasing rubbing strengths. This disagreement can be understood as one follows the experimental situation closely. First of all, we note that in experiments a relatively small order parameter was found in the vicinity of an unrubbed polyimide surface, i.e., $S_{s} \approx 0.2$, in the nematic phase of $8 \mathrm{CB}[3]$. This indicates that there are several nematic domains with a uniform azimuthal orientational distribution that still retain a tilt angle $\theta_{0}$ with respect to the surface [3]. Therefore the unrubbed surface may not affect the bulk

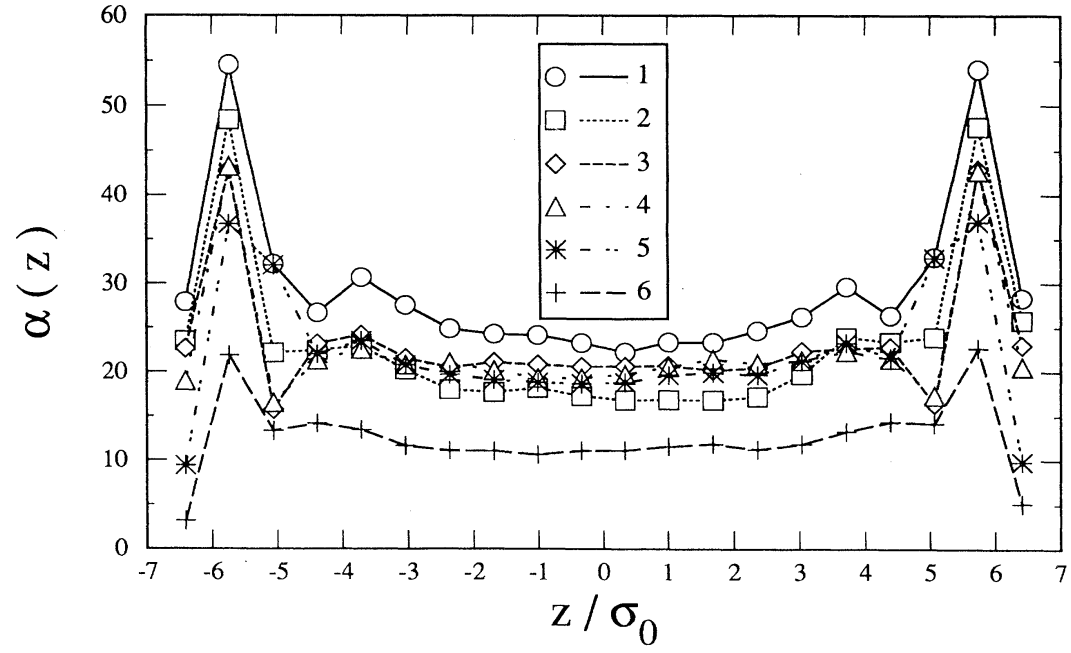

FIG. 3. Tilt angle profiles $\alpha(z)$ in the $z$ direction corresponding to all the cases in Fig. 1. In addition, the data + refer to case 6 in which the intermolecular interactions corresponding to case 5 are weakened by a factor of about 0.1 in the vicinity of the surfaces. $\mathbf{d}_{1}=$ 0. $L_{x}=L_{y}=L_{z}=13.5 \sigma_{0}$. 


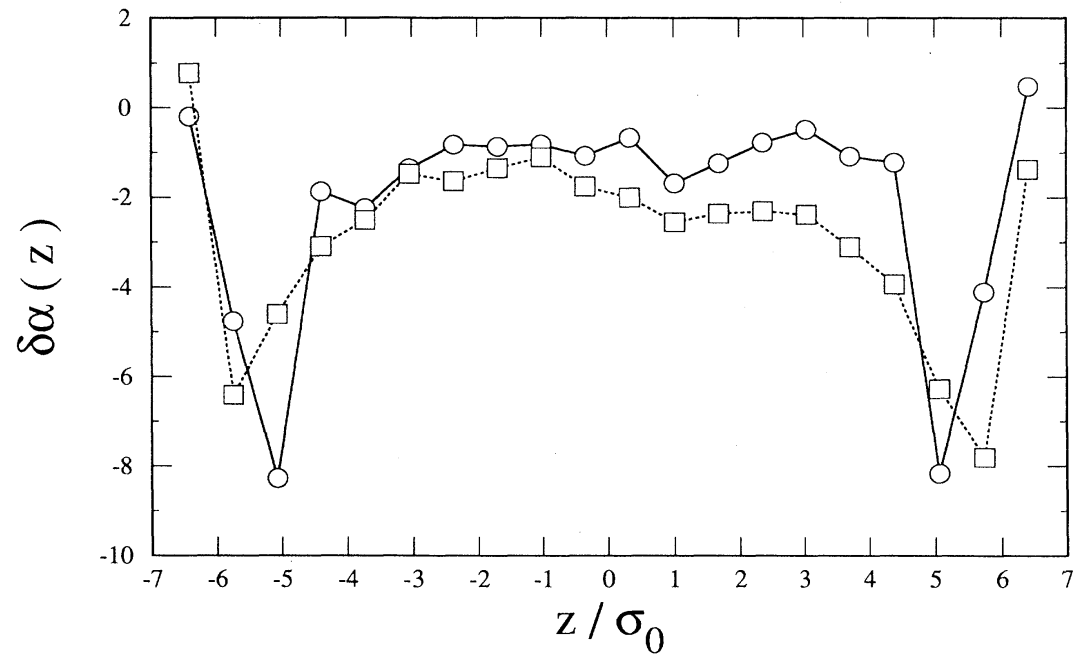

FIG. 4. Deviations of the tilt angles $\delta \alpha(z)$, as a function of $z$, from the values shown in cases 5 and 6 of Fig. 3 at $\mathbf{d}_{1}=2 \hat{\mathbf{x}}$. The data shown as $\circ$ and $\square$ correspond to cases 5 and 6 , respectively. $L_{x}=L_{y}=L_{z}=13.5 \sigma_{0}$. alignment strongly. However, in the weak rubbing limit, a strong magnetic field along the rubbing direction was employed in the experiments to establish the initial bulk alignment with zero pretilt angle [3]. In our finite-size model system it is impossible to have a zero pretilt angle in the nematic phase without introducing unnecessarily complex SM potentials. An increase of the rubbing strength in experiments may then lead to reorientations of the nematic domains with respect to the rubbing direction. This results in an increase in the nematic order in the vicinity of the surface as observed in the experiments [3], thereby giving rise to a nonzero pretilt angle in the bulk. The stronger the rubbing, the larger the pretilt angle corresponding to the increase in the nematic order in the vicinity of the surface. A saturation value of the pretilt angle is expected when the rubbing is strong enough to cause a complete nematic phase in the vicinity of the surface. At this point increasing further the rubbing strength would directly correspond to our simulation studies: this would lead to a decrease in the pretilt angle, as predicted in our simulations. The assumption that the rubbing only affects the azimuthal components of the orientational distributions, as assumed in the analyses of the experimental data, may not be valid in the strong rubbing limit. In fact, the rubbing in our model affects both the polar and azimuthal orientations of the

[1] B. Jêrôme, Rep. Prog. Phys. 54, 391 (1991).

[2] B. Jêrôme et al., Phys. Rev. Lett. 71, 758 (1993).

[3] X. Zhuang, L. Marrucci, and Y. R. Shen, Phys. Rev. Lett. 73, 1513 (1994).

[4] M. M. Telo da Gama, Mol. Phys. 71, 585 (1984); 71, 611 (1984).

[5] J. G. Gay and B. J. Berne, J. Chem. Phys. 74, 3316 (1981).

[6] M. K. Chalam et al., Mol. Sim. 7, 357 (1991).

[7] B. Mehlig, D. W. Heermann, and B. M. Forrest, Phys. Rev. B 45, 679 (1992); S. Duane et al., Phys. Lett. B 195, 216 (1987).

[8] Z. Zhang and A. Chakrabarti (unpublished).

[9] W. A. Steele and G. D. Hasley, J. Phys. Chem. 59, 57 (1955). molecules close to the surface. According to our simulation study, a large pretilt angle can be obtained in order to improve the quality of a supertwisted nematic display cell by treating the surface in such a way that the nematic order on the surfaces can be maintained well.

In summary, we have shown that that the bulk pretilt angle is controlled by the surface through the orientation of the adsorbed liquid crystal monolayer. The SM potential proposed in this work includes the effects of the geometric shape of the GB molecules, which is an important ingredient of the real interactions and is absent in any mean-field potentials. Our model simulation study should therefore be useful for an understanding of the effects of solid surfaces on the properties of the liquid crystals.

This work has been supported by a grant from the National Science Foundation (NSF) (No. DMR-9312596). The computer calculations were carried out under a NSF grant of computer time from the Pittsburgh Supercomputing Center. This work was also supported by the Danish Natural Science and Technical Science Research Councils. One of us (M.J.Z.) wishes to thank the NSERC of Canada for an operating grant and le Fonds FCAR du Québec for both a team and a centre grant.

[10] M. P. Allen and D. J. Tildesley, Computer Simulation of Liquids (Clarendon, Oxford, 1989).

[11] Z. Zhang and A. Chakrabarti, Phys. Rev. E 50, R4290 (1994).

[12] Each data point involves on average about 40 molecules in the case.

[13] P. G. de Gennes, The Physics of Liquid Crystals (Clarendon, Oxford, 1974).

[14] J. Cognard, Mol. Cryst. Liq. Cryst. Suppl. 1, 1 (1982).

[15] To check for possible finite size effects, we also performed a similar numerical simulation study on the larger system with $L_{z}=22.5 \sigma_{0}$ for the same conditions as those in the case represented by the symbol $*$ of Fig. 3 [16]. No finite size effects were found for the results shown in Figs. 1-3.

[16] Z. Zhang (unpublished). 